\title{
Near-Infrared View of the Nuclear Region of NGC 1808
}

\author{
J. K. Kotilainen \\ Tuorla Observatory, University of Turku, Väisäläntie 20, FIN-21500 \\ Piikkiö, Finland
}

\begin{abstract}
We discuss NIR imaging of the starburst galaxy NGC 1808 . The circumnuclear continuum emission shows no morphological change with wavelength and most of it is produced by an evolved bulge population, not by red supergiants or dust. The line emission arises from distinct hot spots. From comparison of $\mathrm{Br} \gamma$ and $\mathrm{H} \alpha$ fluxes, we derive extinction $A_{V}=3-5$ toward the hot spots. From modeling of the line and continuum luminosities, we derive a star-formation rate of $0.1-0.6 M_{\odot} \mathrm{yr}^{-1}$ and $\mathrm{SN}$ rate $\sim 0.4-11 \times 10^{-3} \mathrm{yr}^{-1}$ per hot spot. The age of the burst is $8-17 \mathrm{Myr}$ for the hot spots and $\sim 40 \mathrm{Myr}$ for the nucleus. Finally, evidence for and against hidden Seyfert activity in NGC 1808 is discussed.
\end{abstract}

\section{Introduction}

The nuclear starburst region of the spiral galaxy NGC $1808(D=16.4 \mathrm{Mpc})$ contains a halo superwind, compact radio sources and a molecular ring. Here we discuss high spatial-resolution images of NGC 1808 in the $J H K$ continuum and $\mathrm{Br} \gamma, \mathrm{H}_{2} 2.12 \mu \mathrm{m}$, and [Fe II] $1.64 \mu \mathrm{m}$ lines. For full discussion and interpretation of the results, see Kotilainen et al. (1996).

\section{Results}

The morphology of the near-infrared (NIR) continuum emission does not change from $J$ to $K$, indicating that red supergiants and hot dust do not dominate the NIR emission. The $\mathrm{Br} \gamma, \mathrm{H}_{2},[\mathrm{Fe} \mathrm{II}]$ and $6-\mathrm{cm}$ radio maps show circumnuclear hot spots with differences in the detailed morphology. The extinction toward the line-emitting region, using case $\mathrm{B}$ recombination and the observed $\mathrm{H} \alpha$ and $\mathrm{Br} \gamma$ fluxes, is $A_{V}=3-5$ for the hot spots. For the continuum, comparison of the observed $J-H$ colors to the mean color of normal galaxies yields $A_{V}=0.5-4$, lower than for the emission lines.

\section{Discussion}

\subsection{Star-Formation Activity in NGC 1808}

We used the evolutionary starburst model of Leitherer \& Heckman (1995) to study the star-forming properties of the hot spots. Assuming constant star 
formation with IMF slope 2.35, and upper mass limit $30 M_{\odot}$, we derive a starformation rate (SFR) of $0.1-0.6 M_{\odot} \mathrm{yr}^{-1}$ per hot spot. Using the radio fluxes and spectral indices, we derive supernova (SN) rates of $\leq 0.4-11 \times 10^{-3} \mathrm{yr}^{-1}$ per hot spot. From the SN rates we estimate that the circumnuclear hot spots are 8-17 Myr old, whereas the nucleus is much older, $\sim 40 \mathrm{Myr}$. The Br $\gamma$ equivalent width of the NGC 1808 nucleus is at the lower end of starburst galaxies (Oliva et al. 1995), providing independent support for a decaying nuclear burst. Our SFR value agrees well with those derived by Krabbe et al. (1994) while our SN rates are lower. The ages agree well for the youngest hot spots and the nucleus. Note that if the hot spots were much older than $10 \mathrm{Myr}$, red supergiants should dominate the continuum, whereas they contribute not more than $10 \%$ (TacconiGarman et al. 1996).

\subsection{The Nuclear Component}

While the circumnuclear hot spots have IR line ratios similar to starbursts, the nucleus is similar to Seyferts. This may be due to a lack of hot stars, and imply the presence of an evolved starburst (see above). The line ratios may also result from higher density or dust content in the nuclear ISM, with the ionizing photons being absorbed by dust grains. Alternatively, there may be additional $\mathrm{H}_{2}$ and [FeII] emission from shocked gas. On the other hand, an obscured Seyfert may contribute to the excitation in the nucleus. This possibility was suggested by Véron-Cetty \& Véron (1985) based on broad $\mathrm{H} \alpha$ profiles. Phillips (1993), however, attributed them to the underlying Balmer absorption. Against the Seyfert hypothesis, the nucleus falls onto the correlation between [FeII] and radio emission for starbursts. However, while the soft X-ray emission from NGC 1808 is extended, the hard $\mathrm{X}$-ray source is compact and highly absorbed (Awaki \& Koyama 1993), indicating a Seyfert. Also, the red nuclear colors indicate the presence of hot dust. This is common in Seyfert nuclei (Kotilainen et al. 1992), but in a starburst the dust would be heated by hot stars, while we have argued above for a deficiency of hot stars in the nucleus. Whatever the nature of the nucleus of NGC 1808, there are interesting evolutionary implications. The nuclear starburst may have triggered the circumnuclear burst through superwind shocks. Alternatively, the formation of an obscured Seyfert may be the result of feeding the nucleus by gas driven inward by the starburst.

\section{References}

Awaki, H., \& Koyama, K. 1993, Adv. Space Res., 13, 221.

Kotilainen, J. K., et al. 1992, MNRAS, 256, 149.

Kotilainen, J. K., et al. 1996, A\&A, in press.

Krabbe, A., Sternberg, A., \& Genzel, R. 1994, ApJ, 425, 72.

Leitherer, C., \& Heckman, T. M. 1995, ApJS, 96, 38.

Oliva, E., et al. 1995, A\&A, 301, 55.

Phillips, A.C. 1993, AJ, 105, 486.

Tacconi-Garman, L.E., Sternberg, A., \& Eckart, A. 1996, ApJ, in press.

Véron-Cetty, M.-P., \& Véron, P. 1985, A\&A, 145, 425. 